\title{
Micropatterning of Phase-Segregated Supported Lipid Bilayers and Binary Lipid Phases through Polymer Stencil Lift-off
}

\author{
Yujie Zhu and Jose Moran-Mirabal* \\ Department of Chemistry and Chemical Biology, McMaster University, 1280 Main Street West, \\ Hamilton, Ontario L8S4L8, Canada \\ E-Mail: mirabj@memaster.ca
}

SUPPORTING MATERIAL 

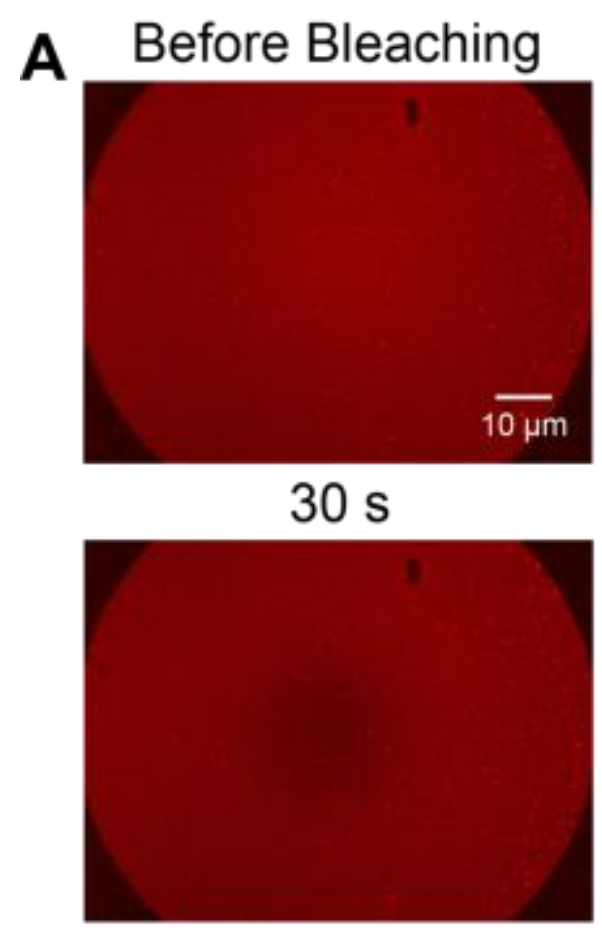

B

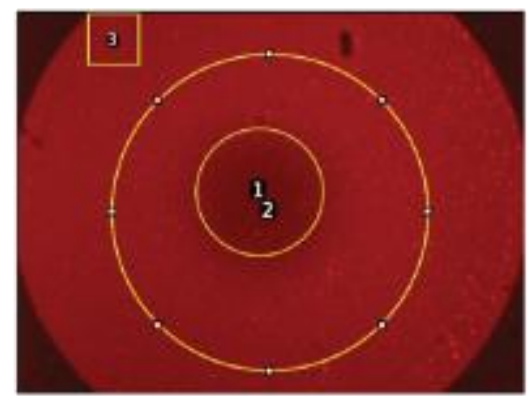

After Bleaching

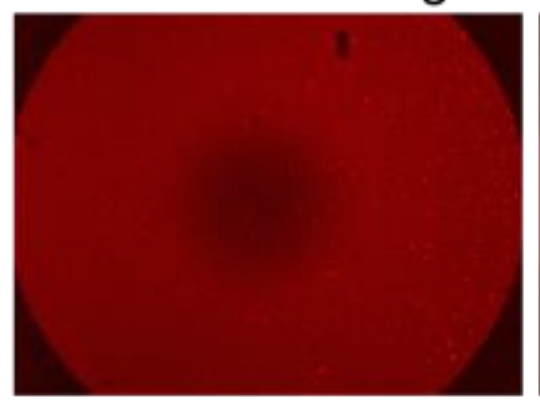

$2 \min$

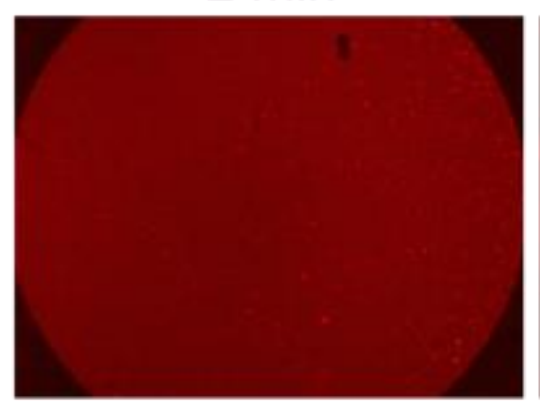

C

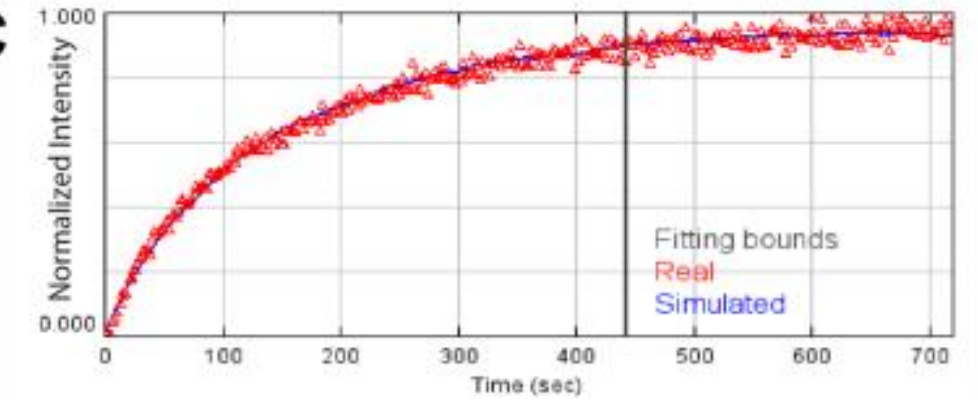

Figure S1. FRAP analysis of homogeneous SLB patterns. (A) Fluorescence images of DOPC:DHPE-LR (99:0.1) SLB pattern shows the center of a circular area being bleached, and the fluorescence recovery over time. (B) The selection of regions of interest are shown for simFRAP analysis using ImageJ software. Area 1 indicates the bleached area, area 2 is the "cell" around the bleached area and area 3 is the reference section used to correct for bleaching. (C) Typical output of the the simFRAP plugin, which gives information of the normalized intensity of the bleached area that is used to calculate the diffusion coefficient. All the images were acquired at the same magnification. 

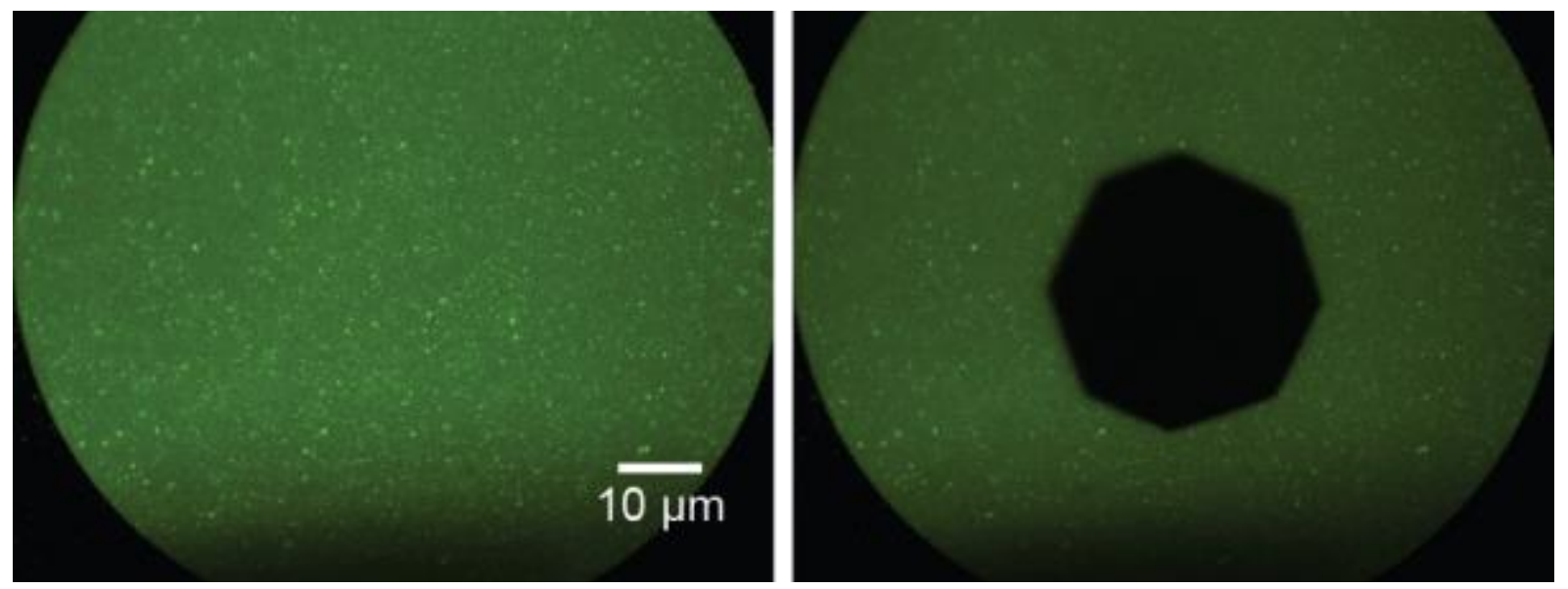

Figure S2. FRAP test of homogeneous DSPC SLB patterns. The DSPC:DHPE-OG (99:1) patterns did not recover after photobleaching at room temperature (picture taken 5 minutes after bleaching). Both images were acquired at the same magnification. 


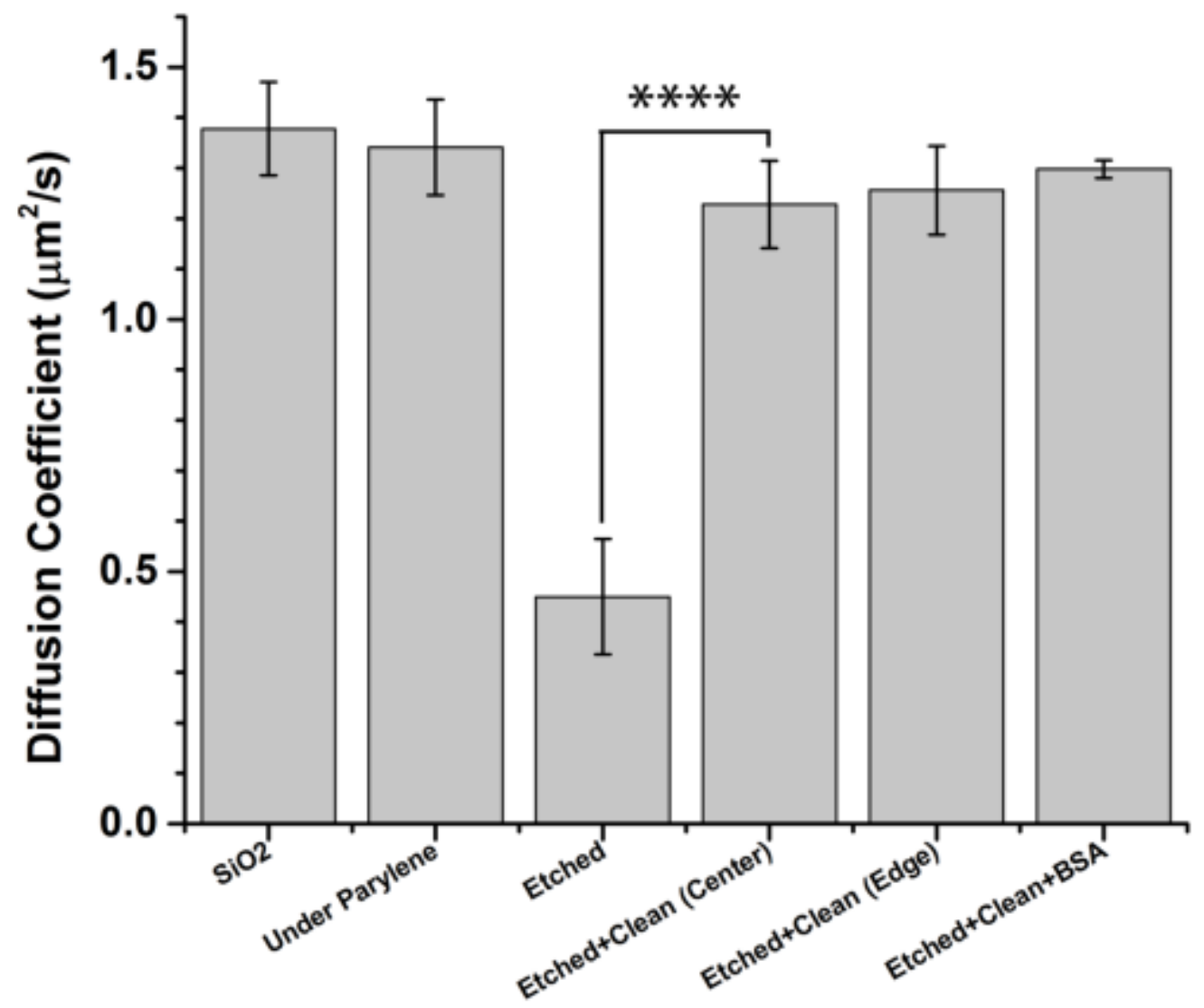

Figure S3. Diffusion coefficients extracted from FRAP measurements on SLBs formed on different substrates. The substrates tested were bare clean $\mathrm{SiO}_{2}$ wafers, wafers where Parylene coating was deposited and then lifted off (Under Parylene), wafers where features were etched into the Parylene and the SLB was formed in the openings before (Etched) and after the $\mathrm{UV} / \mathrm{O}_{3}+$ base cleaning (Etched+Clean), and wafers where the lift-off was performed in buffer containing BSA (Etched+Clean+BSA). The key observations in these experiments were: (1) SLBs formed in the substrates after etching only showed diminished mobility, (2) the UV/O $3+$ base cleaning removed any polymer residue and afforded SLBs with high mobility, comparable to that of piranha cleaned $\mathrm{SiO}_{2}$ substrates $\left(\mathrm{SiO}_{2}\right)$, (3) the mobility at the center and edge of the patterned features is statistically equal, and (4) using BSA during lift-off does not alter the bilayer mobility. Statistical significance evaluated through ANOVA: $* * * * p<0.0001$. 
Without BSA

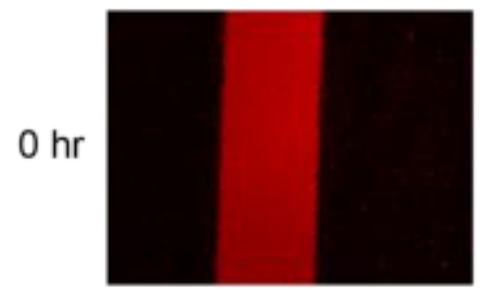

$3 \mathrm{hr}$

$12 \mathrm{hr}$

$6 \mathrm{hr}$


With BSA
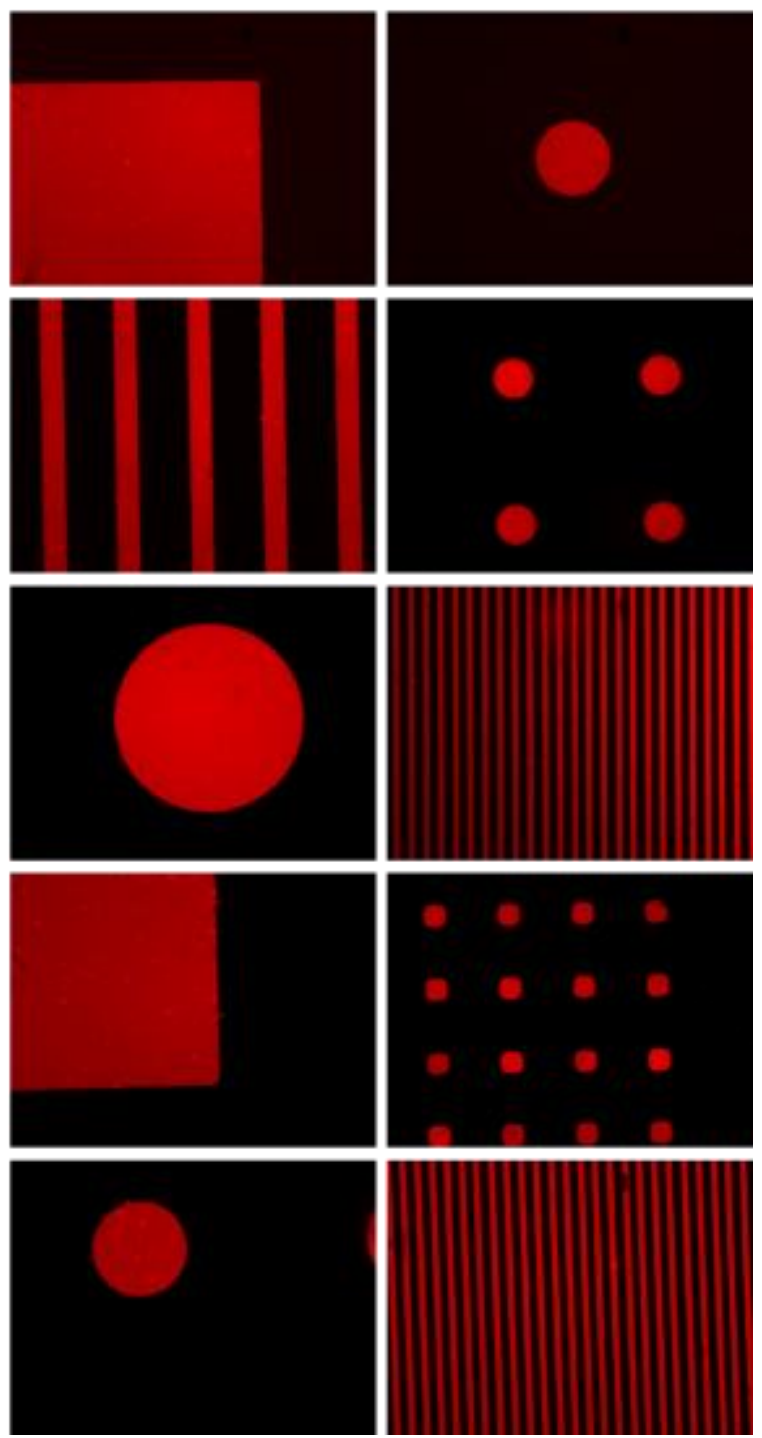

Figure S4. Evaluation of the effect of BSA blocking treatment during polymer lift-off on pattern fidelity. Epifluorescence images show homogeneous DOPC:DHPE-LR (99: 0.1) SLB patterns without (left two columns) and with BSA treatment (right two columns) during lift-off. The SLB patterns were imaged immediately after lift-off, and at 3, 6, 12, and $24 \mathrm{hr}$ after lift-off. It can be seen that without the BSA blocking treatment, the lipid spreads at the edges of the patterned features, while this does not occur when BSA is used. Thus, the BSA blocking treatment during lift-off produces faithful pattern transfer and prevents feature shape degradation due to bilayer spreading. All the images were acquired at the same magnification using the same acquiring settings. 


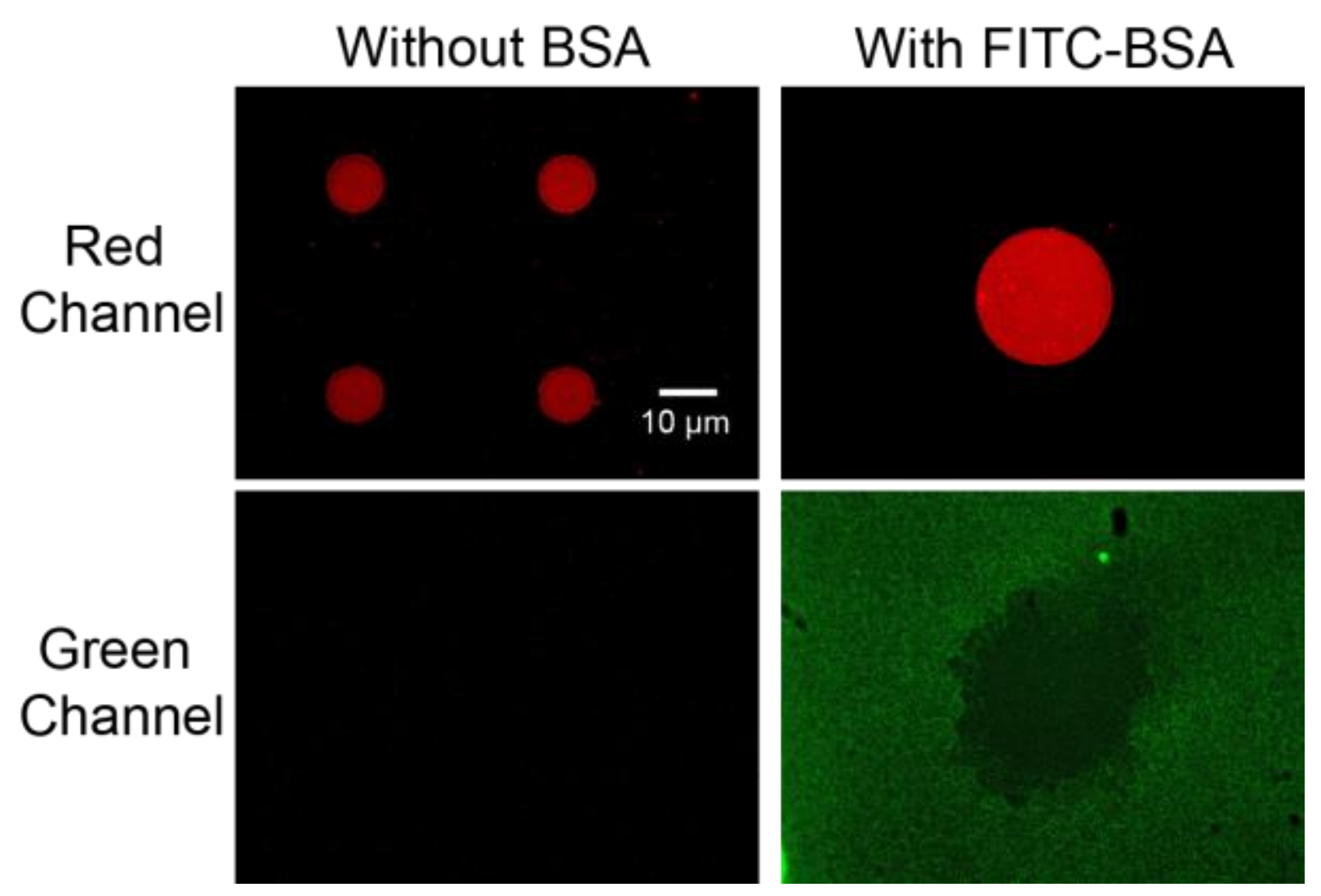

Figure S5. BSA binding on the substrate after polymer lift-off. Epifluorescence images of both Rhodamine and FITC channels were taken for homogeneous DOPC:DHPE-LR (99:0.1) SLB patterns without (left) and with (right) fluorescein labeled BSA used in the blocking treatment. Increased fluorescein intensity in the FITC channel was observed on area outside of the patterns containing the SLBs. The images for same channel were acquired at the same magnification using the same acquisition settings. 

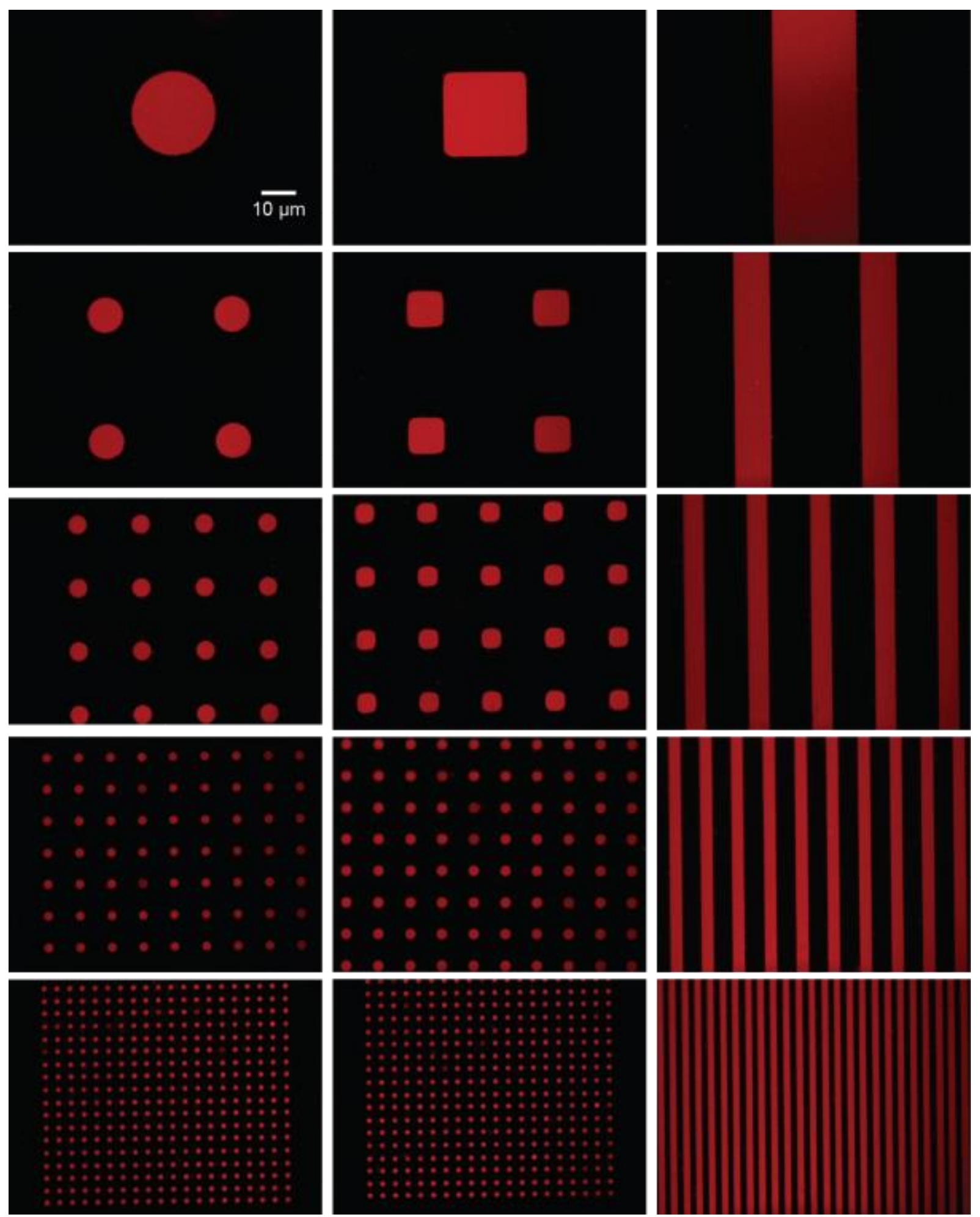

Figure S6. Epifluorescence images show homogeneous DOPC:DHPE-LR (99:1) SLB patterns of various shapes and sizes produced through the polymer stencil lift-off technique using BSA blocking treatment. All images were obtained at the same magnification. 


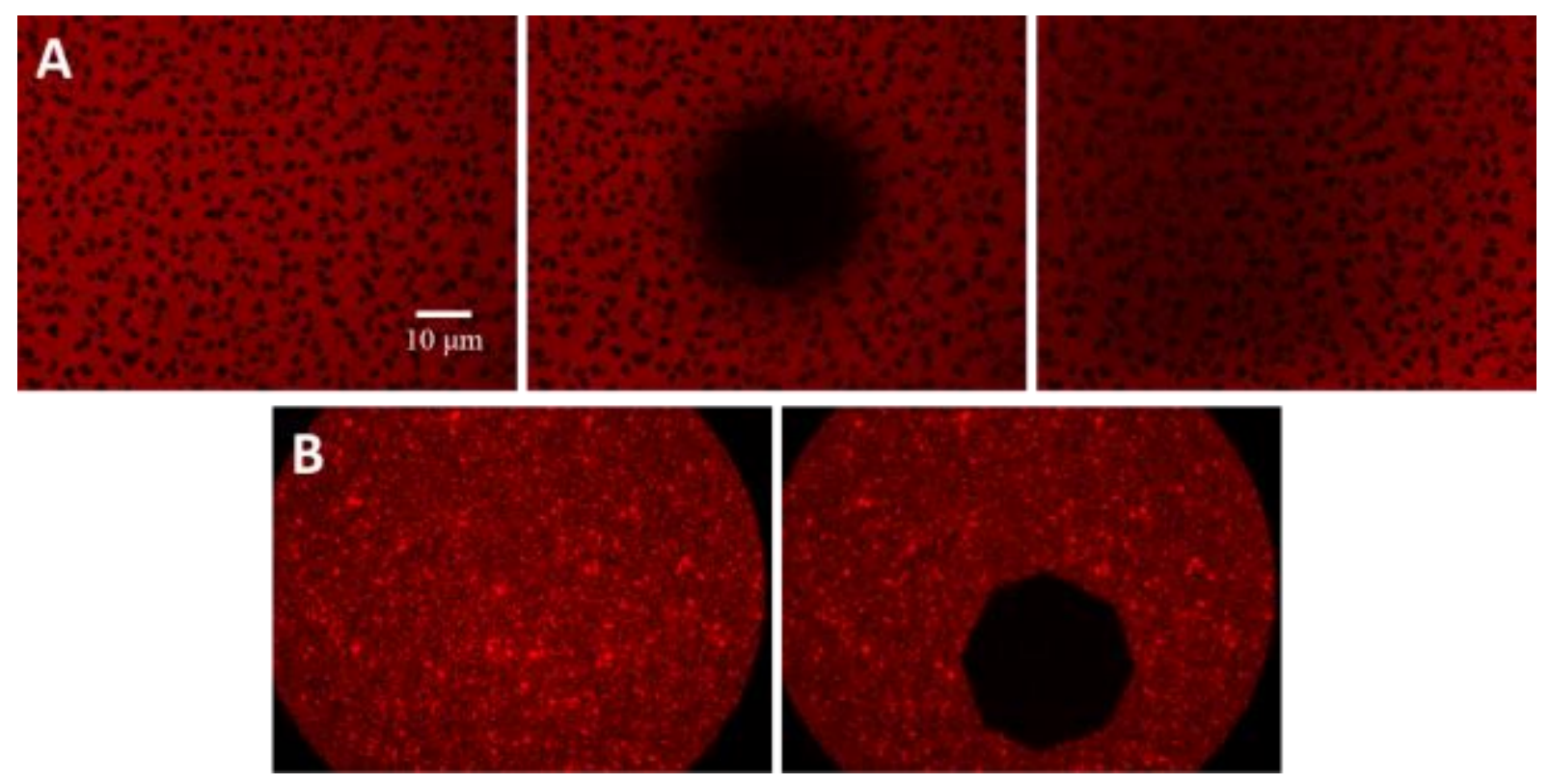

Figure S7. Qualitative bleaching tests of phase-segregating DOPC:DSPC SLB bilayers: (A) Quick fluorescence recovery was observed on DOPC:DSPC:DHPE-LR (49.5:49.5:1) SLBs formed on piranha-cleaned $\mathrm{SiO}_{2}$ wafers; (B) No fluorescence recovery was observed on patterned SLBs with the same composition in substrates where there was no $\mathrm{UV} / \mathrm{O}_{3}$ and base treatment after patterning. All the images were acquired at the same magnification. 

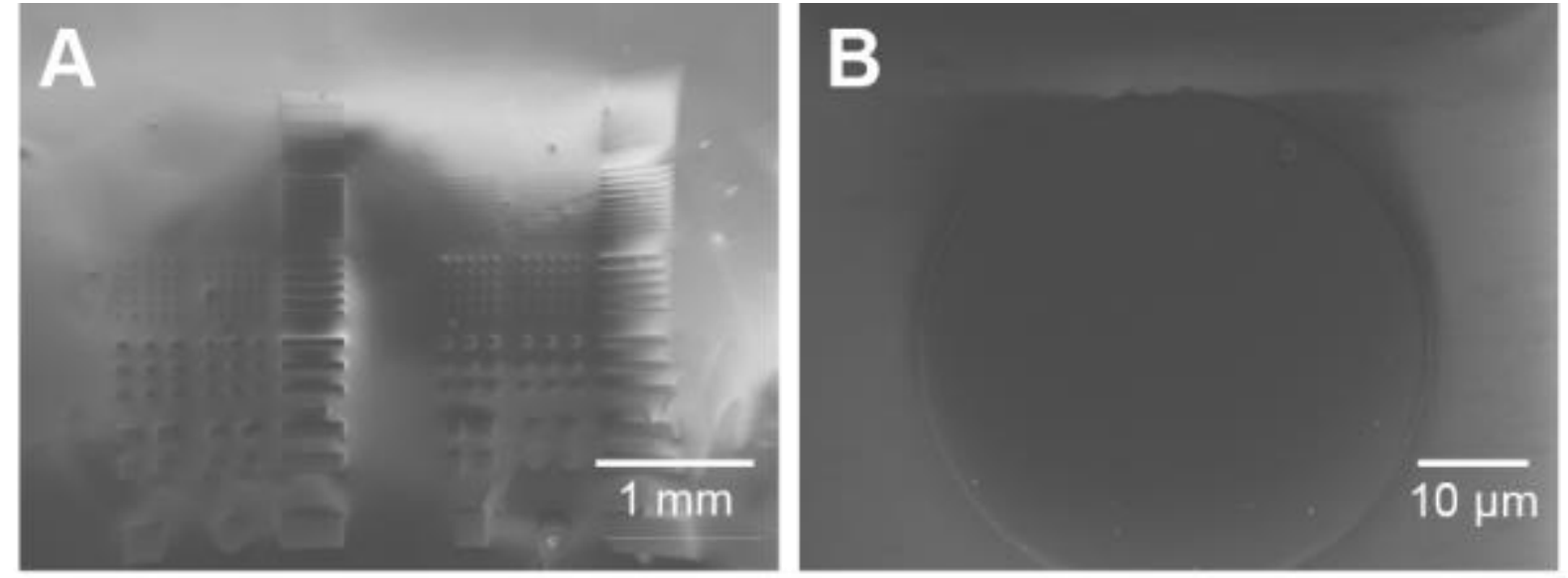

Figure S8. Scanning electron micrographs of $\mathrm{SiO}_{2}$ wafers after Parylene film was patterned and lifted off. (A) Patterned features were visible with SEM. (B) Image obtained within the etched openings show some residual Parylene. 


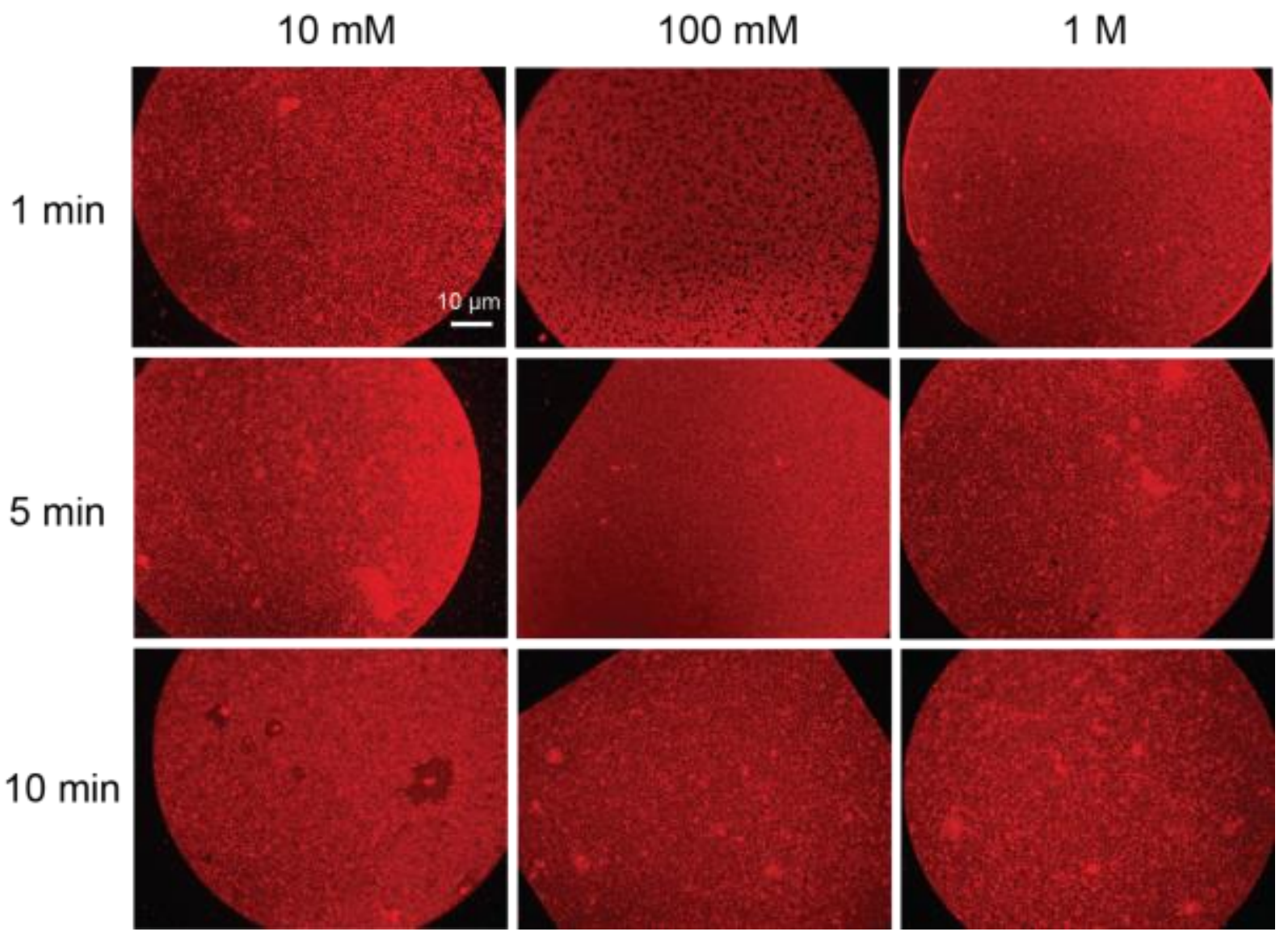

Figure S9. Epifluorescence images showing the phase-segregating DOPC:DSPC SLB patterns on substrates cleaned with a range of $\mathrm{UV} / \mathrm{O}_{3}$ cleaning times (1-10 min) and $\mathrm{NaOH}$ concentrations (0.01-1 M). 


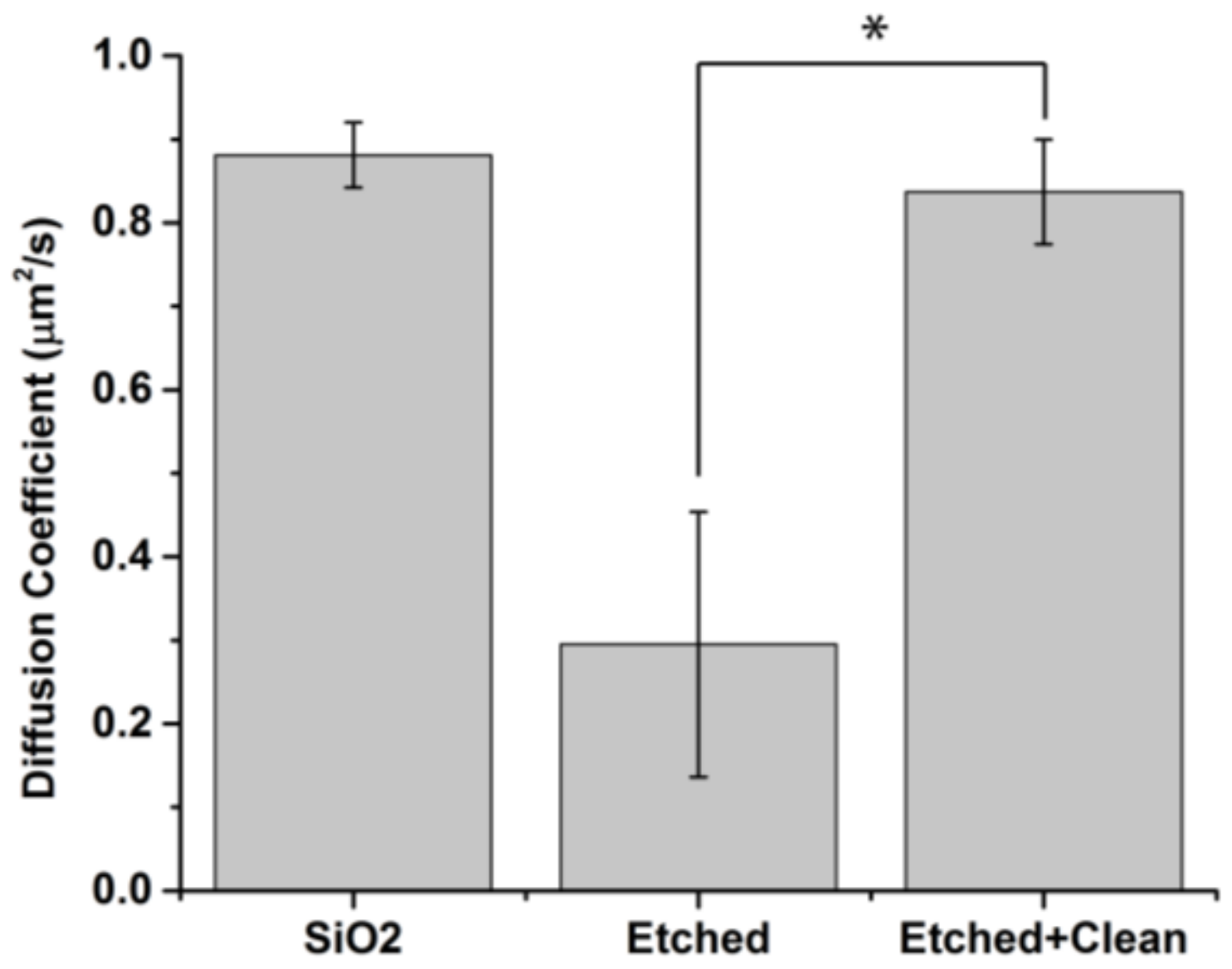

Figure S10. Diffusion coefficients for the lower leaflet of SLBs formed on different substrates were extracted from FRAP measurements performed in the presence of $100 \mathrm{mM} \mathrm{CoCl}_{2}$. The substrates tested were bare clean $\mathrm{SiO}_{2}$ wafers, and wafers where features were etched into the Parylene and the SLB was formed in the openings before (Etched) and after the UV/O $3+$ base cleaning (Etched+Clean). The key observations in these experiments were: (1) The lower leaflet of SLBs formed in the substrates after etching only showed diminished mobility, (2) the $\mathrm{UV} / \mathrm{O}_{3}+$ base cleaning removed any polymer residue and afforded SLBs where the lower leaflet mobility was comparable to that of piranha cleaned $\mathrm{SiO}_{2}$ substrates $\left(\mathrm{SiO}_{2}\right)$. Statistical significance evaluated through ANOVA: $* \mathrm{p}<0.05$. 

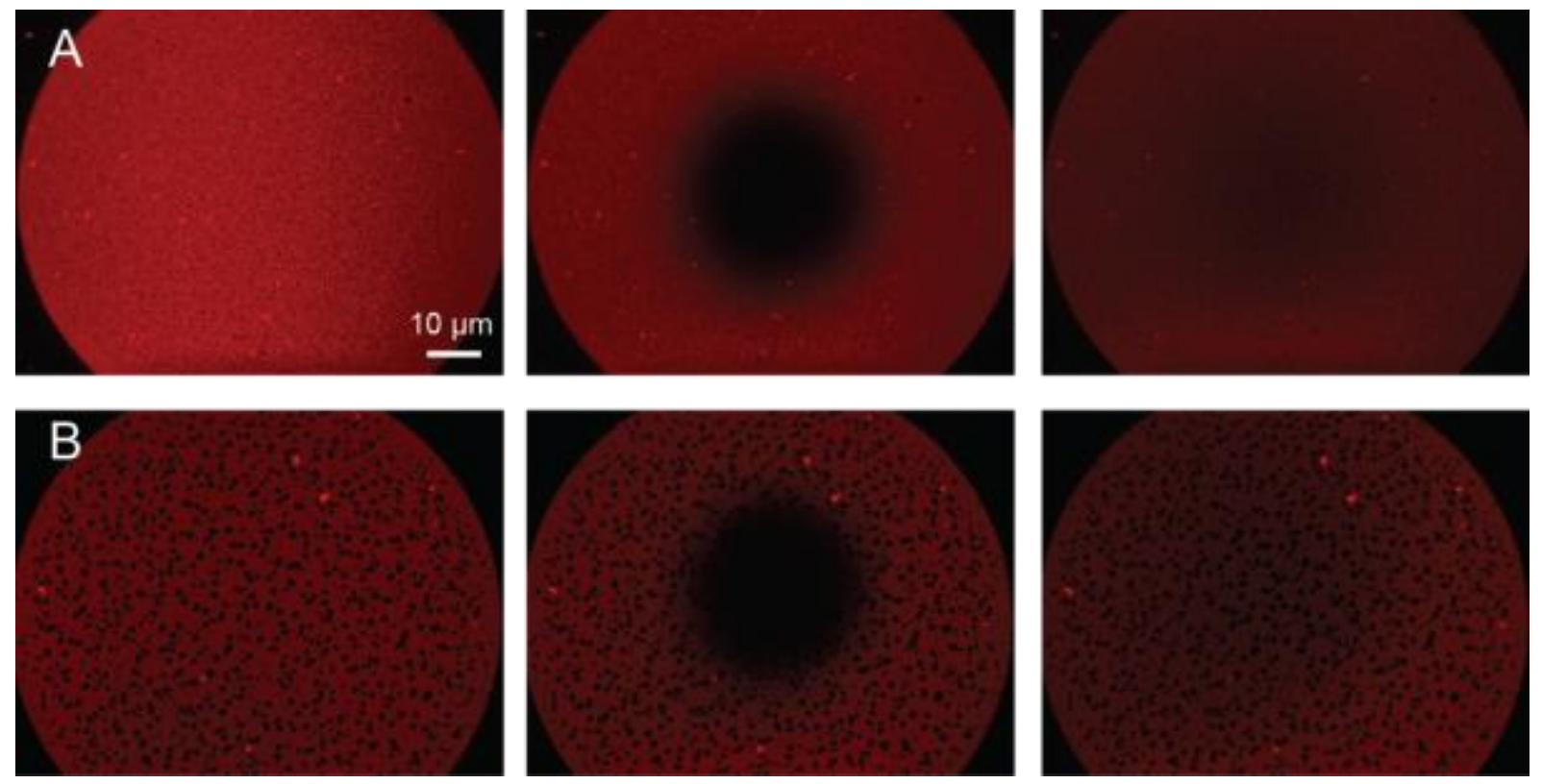

Figure S11. Epifluorescence images showing the cooling effect on phase segregation of patterned DOPC:DSPC:DHPE-LR (49.5:49.5:1) SLBs. Larger gel phase domains were formed when the SLBs were cooled down slowly (B) than when cooled down fast (A). Rapid recovery was observed 5 minutes after bleaching for both cooling conditions, demonstrating that both bilayers were mobile. All the images were acquired at the same magnification. 

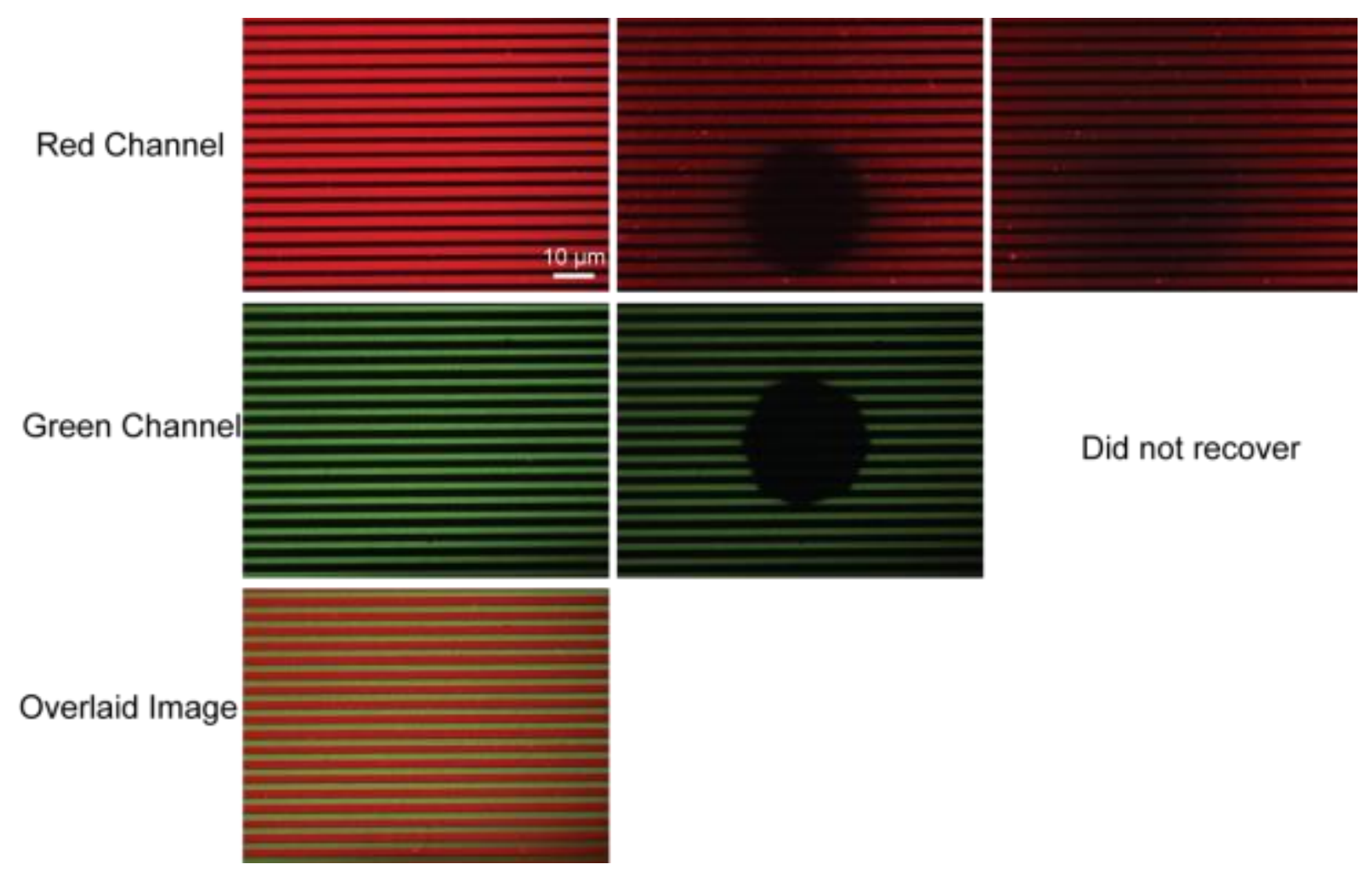

Did not recover

Figure S12. Qualitative fluorescence recovery after bleaching test on binary lipid bilayer patterns with DSPC (green) and DOPC (red) using DHPC-OG and DHPE-LR fluorescent probes, respectively. Overlay image is shown to demonstrate the perfect registration of the two lipid phases. All images were obtained at the same magnification. 

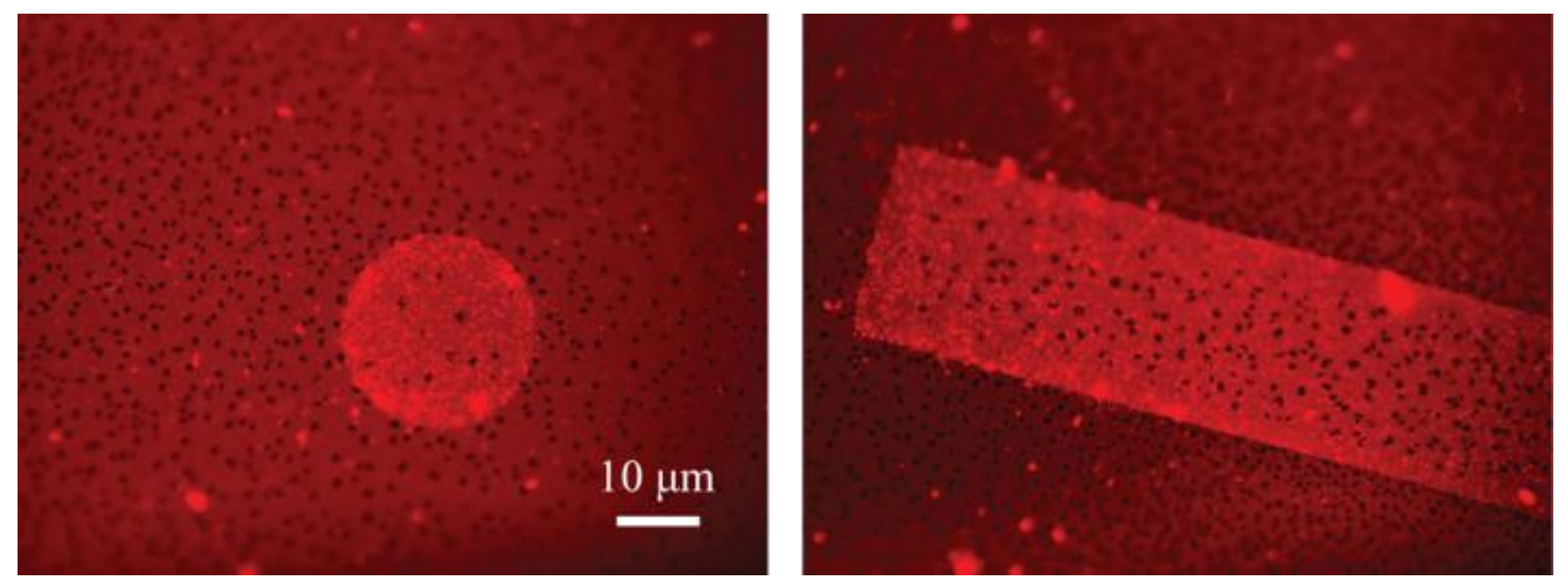

Figure S13. Epifluorescence images of spontaneously phase-segregating SLBs after the binary lipid bilayers patterns were heated up to $60^{\circ} \mathrm{C}$ and cooled down. Both images taken at the same magnification. 